ISSN: 2394-2258

Available at http://scientificadvances.co.in

DOI: http://dx.doi.org/10.18642/ijamml_7100121816

\title{
ON MIXED TOTAL LEAST SQUARES FOR PARAMETER ESTIMATION
}

\author{
Cuiping Guo ${ }^{a}$, Junhuan Peng ${ }^{b}$ and Chuantao Li ${ }^{a}$

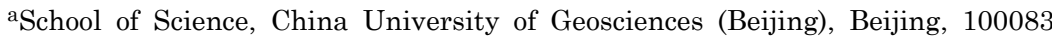 \\ P. R. China \\ ${ }^{b}$ School of Land Science and Technology, China University of Geosciences \\ (Beijing), Beijing, 100083, P. R. China
}

\begin{abstract}
A mixed weighted total least squares (MWTLS) method is presented for an errors-invariables (EIV) model with some fixed columns in the coefficient matrix. An iterative algorithm is derived based on the proposed MWTLS method. Compared with the classical WTLS method, the method represented in this paper improves the computing speed of the estimated parameter, while the fixed columns of the coefficient matrix keeps unperturbed. Furthermore, a simulated example is carried out to demonstrate the performance of the proposed algorithm.
\end{abstract}

Keywords: errors-in-variables, total least squares, mixed weighted total least squares, parameter estimation.

\footnotetext{
${ }^{*}$ Corresponding author.

E-mail address: gcp@cugb.edu.cn (Cuiping Guo).

Copyright ( 2017 Scientific Advances Publishers 2010 Mathematics Subject Classification: 62F10.

Submitted by Jianqiang Gao.

Received February 10, 2017
} 


\section{Introduction}

Consider the linear model

$$
\begin{aligned}
& Y=A \beta+\varepsilon, \\
& \varepsilon \sim\left(0, \sigma^{2} Q\right),
\end{aligned}
$$

where $A \in R^{n \times p}$ is the coefficient matrix with $n>m$, and $Y \in R^{p}$ is the observation vector. It is often assumed in the least square (LS) problem that, the coefficient matrix is exactly known, and only the observation vector has measurement errors. In practice, however, we may encounter the situation in which some or all the elements of the coefficient matrix $A$ are not given deterministically, and they are measured or derived from measurements with random errors. Ignoring such errors in the coefficient matrix usually results in biased estimates of the parameter vector $\beta$, which may cause difficulties and complications in conducting statistical analysis. Models with a random coefficient matrix $A$ have been well known as errors-in-variables (EIV) models (see Fuller [7]; Cheng and Van Ness [5]; Carroll et al. [4]; Buonaccorsi [3]). An appropriate approach to solve EIV models is the total least squares (TLS) method. Now, there are many researches about the total least squares in algorithms such as the singular value decomposition (SVD) algorithm (Golub and Van Loan [8]) and the algorithm based on the Lagrange function (Schaffrin et al. [16, 17]; Fang [6]). For more information about the methodology of TLS, one can refer to Huffel et al. [10, 11]. Recently, TLS has attained much attention. In many applications, such as remote sensing and geodetic datum transformation, data are collected by different instruments with different precisions. Then the ordinary TLS approach is generalized to so called weighted TLS (WTLS) approach where both the observation vector and coefficient matrix have different weight matrices (Schaffrin et al. [16]; Jazaeri et al. [12]). 
In practice, some of the columns of the coefficient matrix may be known exactly. For example, in linear regression with measured independent variables, the elements of the first column of the coefficient matrix is often equal to unity and are surely not random. In system identification, when inputs and outputs of a system are measured with error, and the system is modelled as a transfer function, the coefficient matrix of the function may have exactly known columns. An EIV model with exactly known columns is called a mixed EIV (MEIV) model. Obviously, the computational cost of the MEIV model will increase dramatically according to the traditional solutions of EIV model. For the MEIV model, Golub et al. [9] developed the mixed LS-TLS method by computing a $\mathrm{QR}$ factorization of the known columns and then solved a TLS problem of reduced dimension. And later, this method was extended by Huffel et al. $[10,11]$ to the multi-dimensional case.

In survey engineering, Akyilmaz [1] performed mixed LS-TLS method to solve coordinate transformation problems. Amiri-Simkooei et al. [2] presented the weighted TLS formulation for a MEIV model based on the standard least squares. In this paper, we will provide the mixed weighted total least squared (MWTLS) estimators for the MEIV models by Lagarange-multiplier method. The structure of this paper is as follows. Section 2 presents MWTLS estimators for MEIV models. A simulation study is included in Section 3, and the paper is concluded in Section 4.

\section{Weighted Total Least Squared Estimators for the MEIV Model}

Let the EIV model be defined as follows:

$$
\begin{gathered}
y=\left(A-E_{A}\right) \xi+e_{y}, \\
{\left[\begin{array}{l}
e_{y} \\
e_{A}
\end{array}\right] \sim\left(\left[\begin{array}{l}
0 \\
0
\end{array}\right], \sigma_{o}^{2}\left[\begin{array}{cc}
Q_{y} & 0 \\
0 & Q_{A}
\end{array}\right]\right), P_{y}=Q_{y}^{-1}, P_{A}=Q_{A}^{-1},}
\end{gathered}
$$


where $y$ and $e_{y}$ are the $n \times 1$ observation vector and the corresponding random error vector in (2), respectively. Matrix $A$ and $E_{A}$ are full column-rank $n \times p$ stochastic and the corresponding random error matrix, respectively. Vector $\xi$ is the unknown parameter vector by $n \times 1$. Denote $e_{A}=v e c\left(E_{A}\right)$ ('vec' denotes the operator that stacks one column of a matrix underneath the previous one). The symbol $\sigma_{0}^{2}$ denotes the unknown variance component. Matrices $Q_{y}$ and $P_{y}$ are the cofactor matrix and the weight matrix of the observation vector $y$, and $Q_{A}$ and $P_{A}$ are the cofactor-matrix and the weight matrix of the matrix $e_{A}$. Without loss of generality, we assume that the first $p-t$ columns of the coefficient matrix do not contain errors, but the last $t$ columns of the coefficient matrix do not contain errors. The MEIV model be defined as follows:

$$
\begin{aligned}
& y=A_{1} \beta_{1}+\left(A_{2}-E_{2}\right) \beta_{2}+e_{y}, \\
& {\left[\begin{array}{l}
e_{y} \\
e_{2}
\end{array}\right] \sim\left(\left[\begin{array}{l}
0 \\
0
\end{array}\right], \sigma^{2}\left[\begin{array}{cc}
Q_{y} & 0 \\
0 & Q_{2}
\end{array}\right]\right) .}
\end{aligned}
$$

In (3), $y$ and $e_{y}$ are the $n \times 1$ observation vector and the corresponding random error vector, respectively. Matrices $A_{1}$ is the $n \times(p-t)$ matrix, $A_{2}$ is the $n \times t$ matrix, $E_{2}$ is the corresponding $n \times t$ matrix of random errors, $\beta_{1}$ is the $p-t$ vector of unknown parameters, $\beta_{2}$ is the $t$ vector of unknown parameters, $e_{2}=v e c\left(E_{2}\right)$ ('vec' denotes the operator that stacks one column of a matrix underneath the previous one). The symbol $\sigma_{o}^{2}$ denotes the unknown variance component and $Q_{y}$ is the cofactor matrix of the observation vector $y$, and $Q_{2}$ is the cofactor matrix of $E_{2}$. 
Using vec $(A B C)=\left(C^{T} \otimes A\right) \operatorname{vec}(B)$ (Lütkepohl [13]), the model in (3) can be rewritten as

$$
y=A_{1} \beta_{1}+A_{2} \beta_{2}-\left(\beta_{2}^{T} \otimes I_{n}\right) e_{2}+e_{y},
$$

where $\otimes$ denotes the Kronecker product of matrices.

The weighted total least squares principle is to minimize the objective function

$$
S=e_{y}^{T} Q_{y}^{-1} e_{y}+e_{2}^{T} Q_{2}^{-1} e_{2}
$$

By employing the equivalent target function in accordance with Lagrange, we have

$$
\Phi\left(e_{y}, e_{2}, \lambda, \xi\right)=e_{y}^{T} Q_{y}^{-1} e_{y}+e_{2}^{T} Q_{2}^{-1} e_{2}+2 \lambda^{T}\left[y-A_{1} \beta_{1}-A_{2} \beta_{2}+\left(\beta_{2}^{T} \otimes I_{n}\right) e_{2}-e_{y}\right] .
$$

Then the necessary Euler-Lagrange conditions are derived, namely,

$$
\begin{gathered}
\left.\frac{1}{2} \frac{\partial \Phi}{\partial e_{y}}\right|_{\widetilde{e}_{y}, \widetilde{e}_{2}, \hat{\lambda}, \hat{\beta}_{1}, \hat{\beta}_{2}}=Q_{y}^{-1} \widetilde{e}_{y}-\hat{\lambda}=0, \\
\left.\frac{1}{2} \frac{\partial \Phi}{\partial e_{2}}\right|_{\widetilde{e}_{y}, \widetilde{e}_{2}, \widehat{\lambda}, \hat{\beta}_{1}, \hat{\beta}_{2}}=Q_{2}^{-1} \widetilde{e}_{2}+\left(\hat{\beta}_{2} \otimes I_{n}\right) \hat{\lambda}=0 \\
\left.\frac{1}{2} \frac{\partial \Phi}{\partial \lambda}\right|_{\widetilde{e}_{y}, \widetilde{e}_{2}, \hat{\lambda}, \hat{\beta}_{1}, \hat{\beta}_{2}}=y-A_{1} \hat{\beta}_{1}-A_{2} \hat{\beta}_{2}+\left(\hat{\beta}_{2}^{T} \otimes I_{n}\right) \widetilde{e}_{2}-\widetilde{e}_{y}=0, \\
\left.\frac{1}{2} \frac{\partial \Phi}{\partial \beta_{1}}\right|_{\widetilde{e}_{y}, \widetilde{e}_{2}, \hat{\lambda}, \hat{\beta}_{1}, \hat{\beta}_{2}}=-A_{1}^{T} \hat{\lambda}=0 \\
\left.\frac{1}{2} \frac{\partial \Phi}{\partial \beta_{2}}\right|_{\widetilde{e}_{y}, \widetilde{e}_{2}, \hat{\lambda}, \hat{\beta}_{1}, \hat{\beta}_{2}}=-A_{2}^{T} \hat{\lambda}+\widetilde{E}_{2}^{T} \hat{\lambda}=0
\end{gathered}
$$

where tildas indicate "predicted" vectors, and hats indicate "estimated" ones. Now, $\tilde{e}_{y}$ and $\tilde{e}_{2}$ can be expressed in terms of $\hat{\lambda}$ by using (5) and (6). This leads to 


$$
\begin{gathered}
\tilde{e}_{y}=Q_{y} \hat{\lambda}, \\
\tilde{e}_{2}=-Q_{2}\left(\hat{\beta}_{2} \otimes I_{n}\right) \hat{\lambda},
\end{gathered}
$$

and after inserting this into (7), we obtain

$$
\hat{\lambda}=\left[Q_{y}+\left(\hat{\beta}_{2}^{T} \otimes I_{n}\right) Q_{2}\left(\hat{\beta}_{2} \otimes I_{n}\right)\right]^{-1}\left(y-A_{1} \hat{\beta}_{1}-A_{2} \hat{\beta}_{2}\right) .
$$

Let

$$
Q_{1}=Q_{y}+\left(\hat{\beta}_{2}^{T} \otimes I_{n}\right) Q_{2}\left(\hat{\beta}_{2} \otimes I_{n}\right),
$$

where $Q_{1}$ is invertible. We readily obtain

$$
\hat{\lambda}=Q_{1}^{-1}\left(y-A_{1} \hat{\beta}_{1}-A_{2} \hat{\beta}_{2}\right) .
$$

Inserting (14) into (8), we get

$$
\hat{\beta}_{1}=\left(A_{1}^{T} Q_{1}^{-1} A_{1}\right)^{-1} A_{1}^{T} Q_{1}^{-1}\left(y-A_{2} \hat{\beta}_{2}\right) .
$$

Let

$$
Q_{3}=A_{2}^{T} Q_{1}^{-1}\left[I_{n}-A_{1}\left(A_{1}^{T} Q_{1}^{-1} A_{1}\right)^{-1} A_{1}^{T} Q_{1}^{-1}\right]
$$

and using

$$
\widetilde{E}_{2}^{T} \hat{\lambda}=\left(I_{t} \otimes \hat{\lambda}^{T}\right) \widetilde{e}_{2},
$$

we obtain from (9) that

$$
\hat{\beta}_{2}=\left(Q_{3} A_{2}\right)^{-1}\left[Q_{3} y-\left(I_{t} \otimes \hat{\lambda}^{T}\right) \widetilde{e}_{2}\right] .
$$

Inserting (17) into (15), the closed-form expression of the estimated parameter vector $\hat{\beta}_{1}$ and $\hat{\beta}_{2}$ can be derived as

$$
\left\{\begin{array}{l}
\hat{\beta}_{1}=\left(A_{1}^{T} Q_{1}^{-1} A_{1}\right)^{-1} A_{1}^{T} Q_{1}^{-1}\left\{\left[I-A_{2}\left(Q_{3} A_{2}\right)^{-1}\right] y+A_{2}\left(Q_{3} A_{2}\right)^{-1}\left(I_{t} \otimes \hat{\lambda}^{T}\right) \widetilde{e}_{2}\right\}, \\
\hat{\beta}_{2}=\left(Q_{3} A_{2}\right)^{-1}\left[Q_{3} y-\left(I_{t} \otimes \hat{\lambda}^{T}\right) \tilde{e}_{2}\right] .
\end{array}\right.
$$


In the analogy with the standard least squares, the estimator of the variance component $\sigma^{2}$ is given as

$$
\hat{\sigma}_{0}^{2}=\frac{e_{y}^{T} Q_{y}^{-1} e_{y}+e_{2}^{T} Q_{2}^{-1} e_{2}}{n-p} .
$$

Substitution of $\tilde{e}_{y}$ from (10) and $e_{2}$ from Equation (11) into (19), yields

$$
\hat{\sigma}_{0}^{2}=\frac{\hat{\lambda}^{T}\left[Q_{y}+\left(\hat{\beta}_{2}^{T} \otimes I_{n}\right) Q_{2}\left(\hat{\beta}_{2} \otimes I_{n}\right)\right] \hat{\lambda}}{n-p} .
$$

From (13), $\hat{\sigma}_{0}^{2}$ can be obtained as

$$
\hat{\sigma}_{0}^{2}=\frac{\hat{\lambda}^{T}\left(y-A_{1} \hat{\beta}_{1}-A_{2} \hat{\beta}_{2}\right)}{n-p} .
$$

After giving an initial value of the parameter vector, we compute the solution with (18) iteratively. Note that, in each iteration, the matrices and vectors containing the parameter vector and the error matrix should be updated. Therefore, the MWTLS procedure for parameter estimation is summarized as follows:

\section{MWTLS Algorithm:}

Step 1. Set $\left(\begin{array}{l}\hat{\beta}_{1}^{0} \\ \hat{\beta}_{2}^{0}\end{array}\right)=\left(\left(A_{1}, A_{2}\right)^{T} P_{y}\left(\begin{array}{c}A_{1} \\ A_{2}\end{array}\right)\right)^{-1}\left(A_{1}, A_{2}\right)^{T} y$.

Step 2. For $i \in \mathbb{N}$, compute

$$
\begin{array}{r}
Q_{1}^{i}=Q_{y}+\left(\hat{\beta}_{2}^{i T} \otimes I_{n}\right) Q_{2}\left(\hat{\beta}_{2}^{i} \otimes I_{n}\right), Q_{3}^{i}=A_{2}^{T}\left(Q_{1}^{i}\right)^{-1}\left\{I_{n}-A_{1}\left[A_{1}^{T}\left(Q_{1}^{i}\right)^{-1} A_{1}\right]^{-1} A_{1}^{T}\left(Q_{1}^{i}\right)^{-1}\right\}, \\
\hat{\lambda}^{i}=\left(Q_{1}^{i}\right)^{-1}\left(y-A_{1} \hat{\beta}_{1}^{i}-A_{2} \hat{\beta}_{2}^{i}\right),\left(\widetilde{e}_{2}\right)^{i}=-Q_{2}\left(\hat{\beta}_{2}^{i} \otimes I_{n}\right) \hat{\lambda}^{i}, \hat{\beta}_{2}^{i}=\left(Q_{3}^{i} A_{2}\right)^{-1} \\
{\left[Q_{3}^{i} y-\left(I_{t} \otimes\left(\hat{\lambda}^{i}\right)^{T}\right) \tilde{e}_{2}^{i}\right] .}
\end{array}
$$

Step 3. Stop when $\left\|\hat{\beta}_{1}^{i}-\hat{\beta}_{1}^{i-1}\right\|<\varepsilon$ and $\left\|\hat{\beta}_{2}^{i}-\hat{\beta}_{2}^{i-1}\right\|<\varepsilon$ for a chosen threshold $\varepsilon$. 


\section{Numerical Examples}

In order to demonstrate the performance of Algorithm 1, in this section, the algorithm will be applied to a straight line fitting problem representing the MEIV problem, compared with the general WLS algorithm and the algorithm proposed by Fang [6]. The observed data and their corresponding weights are listed in Table 1. We try to estimate the slope $a$ and the intercept $b$ of the regression line

$$
y_{i}-e_{y_{i}}=a \cdot\left(x_{i}-e_{x_{i}}\right)+b
$$

using the MWTLS algorithm above.

Table 1. Observed data $\left(x_{i}, y_{i}\right)$ and corresponding weights, taken from Neri et al. [14]

\begin{tabular}{|c|c|c|c|c|}
\hline$i$ & $x_{i}$ & $W_{x_{i}}$ & $y_{i}$ & $W_{y_{i}}$ \\
\hline 1 & 0.0 & 1000.0 & 5.9 & 1.0 \\
\hline 2 & 0.9 & 1000.0 & 5.4 & 1.8 \\
\hline 3 & 1.8 & 500.0 & 4.4 & 4.0 \\
\hline 4 & 2.6 & 800.0 & 4.6 & 8.0 \\
\hline 5 & 3.3 & 200.0 & 3.5 & 20.0 \\
\hline 6 & 4.4 & 80.0 & 3.7 & 20.0 \\
\hline 7 & 5.2 & 60.0 & 2.8 & 70.0 \\
\hline 8 & 6.1 & 20.0 & 2.8 & 70.0 \\
\hline 9 & 6.5 & 1.8 & 2.4 & 100.0 \\
\hline 10 & 7.4 & 1.0 & 1.5 & 500.0 \\
\hline
\end{tabular}


Modifying (22) as follows:

$$
\left(\begin{array}{c}
y_{1} \\
y_{2} \\
\vdots \\
y_{n}
\end{array}\right)=\left(\begin{array}{c}
1 \\
1 \\
\vdots \\
1
\end{array}\right) \cdot b+\left[\left(\begin{array}{c}
x_{1} \\
x_{2} \\
\vdots \\
x_{n}
\end{array}\right)-\left(\begin{array}{c}
e_{x_{1}} \\
e_{x_{2}} \\
\vdots \\
e_{x_{n}}
\end{array}\right)\right] \cdot a,
$$

where (23) is a typical MEIV model and * means that there is no result about the estimator of the variance component in Neri's paper.

Table 2. Results of straight line fit to observed data of Table 1

\begin{tabular}{|c|c|c|c|c|}
\hline $\begin{array}{c}\text { Parameter } \\
\text { estimate }\end{array}$ & $\begin{array}{c}\text { Exact solution } \\
\text { (Neri et al.) }\end{array}$ & WLS & $\begin{array}{c}\text { WTLS } \\
\text { (Schaffrin et al.) }\end{array}$ & $\begin{array}{c}\text { MTLS } \\
\text { (this paper) }\end{array}$ \\
\hline$a$ & -0.480533407 & -0.610812956 & -0.480533407 & -0.480533407 \\
\hline$b$ & 5.47991022 & 6.100109317 & 5.479910224 & 5.479910224 \\
\hline$\hat{\sigma}^{2}$ & $*$ & 4.29315094 & 1.48329415 & 1.48329415 \\
\hline Cpu time & $*$ & 0.0468 & 0.0624 & $\approx 0$ \\
\hline
\end{tabular}

The threshold $\left(\varepsilon=10^{-10}\right)$ has been chosen such as to allow a comparison of our MTLS to the "exact solution" reported by Neri et al. [14] and the solution reported by Schaffrin and Wieser [17]. In fact, the results indicate that the estimated line parameters are the same and coincide with the exact solutions as reported by Neri et al. [14] and Schaffrin and Wieser [17]. Therefore, it is possible to find exact results for a regression line analysis affected by errors, without requiring any kind of approximation. Compared with WTLS, the MTLS algorithm presented in this paper consumes little time, therefore, the computation speed is accelerated. In fact, if the coefficient matrix has more fixed columns, the MTLS algorithm can present its superiority over the TLS algorithm. 


\section{Conclusion}

For a MEIV model, considering the random errors may exist in both of the observation vector and the coefficient matrix, and the coefficient matrix have some fixed columns, the MWTLS algorithm is constructed in this paper. A numerical example is carried out to demonstrate the performance of the MWTLS compared with WTLS. When all the columns of the coefficient matrix are fixed, the MTLS solution reduces to the ordinary LS estimate. When all the columns of the coefficient matrix are random, the MTLS solution becomes the TLS solution. So, it will have a more wide range of application.

\section{Acknowledgement}

The research is supported by the Fundamental Research Funds for Central Universities (No. 2652015193), Undergraduate Teaching-Reform Project (No. JGYB201529), Graduate Teaching-Reform Project of China of University of Geosciences (Beijing), NSFC (41374016, 41104025 and 41330634), and SKLGED2013-4-8-e.

\section{References}

[1] O. Akyilmaz, Total least squares solution of coordinate transformation, Survey Review 39(303) (2007), 68-80.

[2] A. R. Amiri-Simkooei, S. Mortazavi and J. Asgari, Weighted total least squares applied to mixed observation model, Survey Review 48(349) (2016), 278-286.

[3] J. P. Buonaccorsi, Measurement Error: Models, Methods, and Applications, CRC Press, Boca Raton, 2010.

[4] R. J. Carroll, D. Ruppert, L. A. Stefanski and C. M. Crainiceanu, Measurement Error in Nonlinear Models: A Modern Perspective, CRC Press, 2006.

[5] C. L. Cheng and J. W. Van Ness, Statistical Regression with Measurement Error, Arnold and New York: Oxford University Press, London, 1999.

[6] X. Fang, Weighted total least squares: necessary and sufficient conditions, fixed and random parameters, Journal of Geodesy 87(8) (2013), 733-749.

[7] W. A. Fuller, Measurement Error Models, John Wiley \& Sons, New York, 2009.

[8] G. H. Golub and C. F. Van Loan, An analysis of the total least squares problem, SIAM Journal on Numerical Analysis 17(6) (1980), 883-893. 
[9] G. H. Golub, A. Hoffman and G. W. Stewart, A generalization of the Eckart-YoungMirsky matrix approximation theorem, Linear Algebra and its Applications 88-89(3) (1987), 317-327.

[10] S. V. Huffel and J. Vandewalle, The Total Least Squares Problem: Computational Aspects and Analysis, Society for Industrial and Applied Mathematics, Philadelphia, 1997.

[11] S. V. Huffel, C. L. Cheng, N. Mastronardi, C. Paige and A. Kukush, Total Least Squares and Errors-in-Variables Modeling: Analysis, Algorithms and Applications, Kluwer Academic Publishers, 2002.

[12] S. Jazaeri, A. R. Amiri-Simkooei and M. A. Sharifi, Iterative algorithm for weighted total least squares adjustment, Survey Review 46(334) (2014), 19-27.

[13] H. Lütkepohl, Handbook of Matrices, Wiley, 1996.

[14] F. Neri, G. Saitta and S. Chiofalo, An accurate and straightforward approach to line regression analysis of error-affected experimental data, Journal of Physics E Scientific Instruments 22(22) (1989), 215.

[15] G. Pan, Y. Zhou, H. Sun and W. Guo, Linear observation based total least squares, Survey Review 47(340) (2015), 18-27.

[16] B. Schaffrin, I. Lee, Y. Felus and Y. Choi, Total least-squares for geodetic straightline and plane adjustment, Bollettino Di Geodesia E Scienze Affini 65(3) (2006), 141-168.

[17] B. Schaffrin and A. Wieser, On weighted total least-squares adjustment for linear regression, Journal of Geodesy 82(7) (2008), 415-421. 